\title{
Nonlinear Pressure Dependence of the Interaction Potential of Dense Protein Solutions
}

\author{
Martin A. Schroer, ${ }^{1}$ Jonas Markgraf, ${ }^{2}$ D. C. Florian Wieland, ${ }^{1}$ Christoph J. Sahle, ${ }^{1}$ Johannes Möller, ${ }^{1}$ \\ Michael Paulus, ${ }^{1}$ Metin Tolan, ${ }^{1}$ and Roland Winter ${ }^{2}$ \\ ${ }^{1}$ Faculty of Physics/DELTA, TU Dortmund, Maria-Goeppert-Mayer-Str. 2, 44227 Dortmund, Germany \\ ${ }^{2}$ Faculty of Chemistry, Physical Chemistry-Biophysical Chemistry, TU Dortmund, Otto-Hahn Str. 6, 44227 Dortmund, Germany
}

(Received 7 September 2010; published 26 April 2011)

\begin{abstract}
The influence of pressure on the structure and protein-protein interaction potential of dense protein solutions was studied and analyzed using small-angle x-ray scattering in combination with a liquid state theoretical approach. The structural as well as the interaction parameters of dense lysozyme solutions are affected by pressure in a nonlinear way. The structural properties of water lead to a modification of the protein-protein interactions below 4 kbar, which might have significant consequences for the stability of proteins in extreme natural environments.
\end{abstract}

DOI: 10.1103/PhysRevLett.106.178102

PACS numbers: 87.15.km, 61.05.cf, 81.40.Vw

One of the first studies of the effect of pressure on proteins dates back to 1914, when Nobel laureate P.W. Bridgman discovered that pressures of several kbar are able to unfold albumin under ambient temperature conditions [1]. Since then, a number of studies have been carried out to investigate the temperature-pressure stability diagram of proteins [2-5]. Biophysical studies of pressure effects on proteins have mainly been performed at dilute solution conditions and generally not under conditions where molecular crowding is prevailing, such as observed in cellular environments. Here we report on the effect of pressure on the structure and intermolecular interaction potential of dense protein solutions revealed by small-angle $\mathrm{x}$-ray scattering (SAXS), which seem to be influenced by the particular properties of water at high pressures.

Pressure is a fundamental thermodynamic variable that, next to temperature and the chemical potential of the solute, controls the conformational properties and hence function of biomolecules. Changing the temperature of a system at atmospheric pressure produces a simultaneous change in thermal energy and density. To separate thermal and density effects, one has to perform pressure dependent experiments [2-5]. Because noncovalent interactions play a primary role in the stabilization of biochemical systems, the use of pressure allows one also to change, in a controlled and reversible way, the intermolecular interactions without the major perturbations produced by changes in temperature and/or chemical composition. Moreover, hydrostatic pressures are also relevant for understanding life under extreme conditions, as for instance in exobiological or deep sea environments (where organisms have to cope with pressures up to $\sim 1 \mathrm{kbar}$ ). Finally, the highpressure phase behavior of biomolecules is of significant biotechnological interest (e.g., for high-pressure food processing) [2,3].

Biological macromolecules such as proteins have evolved and function within intracellular environments that are highly crowded. Such macromolecular crowding results in surprisingly large effects on both the equilibria and rates of interactions, including the stability and folding rate of proteins [6]. Quantitative data are largely unknown, however, in particular, under high-pressure conditions [5-7]. By pressure modulation, intermolecular distances can be fine tuned to explore details of the interactions governing the spatial arrangement of dense protein solutions. The properties of water may also be important for the interactions in dense protein solutions, where only a few water layers separate neighboring protein molecules. On these grounds, we performed high-pressure SAXS measurements on dense lysozyme solutions. The SAXS technique allowed us to accurately monitor structural alterations of the protein solution as well as to gain quantitative information on state-dependent protein-protein interactions [8-10].

Lysozyme (14.3 $\mathrm{kDa}, p \mathrm{I}=11$, from hen egg white, Roche GmbH, Mannheim) was dissolved in $25 \mathrm{mM}$ bisTris buffer solution in order to keep the $p \mathrm{H}$ value constant at $p \mathrm{H} 7$ at high pressures [11]. A protein concentration of $c_{p}=100 \mathrm{mg} \mathrm{mL}^{-1}(\sim 10 \mathrm{wt} \%)$ was used except for the determination of the form factor of the protein where a $4.5 \mathrm{mg} \mathrm{mL} \mathrm{m}^{-1}$ solution was applied. To generate highpressure conditions, our custom-built high-pressure cell employing two flat diamond windows was used [12]. A pressure range from 1 to 4000 bar was covered, and the sample temperature was set to 5,15 , and $25^{\circ} \mathrm{C}$. The SAXS measurements were performed at beam lines ID02, ESRF, Grenoble, and at BW4, HASYLAB, Hamburg. The SAXS profiles obtained were background corrected by subtraction of the corresponding buffer signal.

For concentrated protein solutions, the x-ray scattering can be described in the so-called decoupling approximation as the product of the form factor $P(q)$ and an effective structure factor $S_{\text {eff }}(q)(q=(4 \pi / \lambda) \sin (\Theta / 2)$ being the wave vector transfer, $\lambda$ the wavelength, and $\Theta$ the scattering angle), which is related to the intermolecular structure factor $S(q)$ via 


$$
S_{\text {eff }}(q)=1+\frac{\langle F(q)\rangle_{\Omega}^{2}}{P(q)}(S(q)-1)
$$

where $\langle F(q)\rangle_{\Omega}$ is the spherical average of the Fourier transform of the protein's electron density [13]. Applying a statistical mechanical model approach, measurements of $S(q)$ allow extraction of the intermolecular protein-protein interaction potential through the direct correlation function $c(r)$. Here, we used the DLVO (Derjaguin-LandauVerwey-Overbeek) potential and the mean spherical approximation (MSA), i.e., $c(r \geq \sigma)=-V(r) /\left(k_{B} T\right)$, $g(r<\sigma)=0[14,15]$, for linking $S(q)$ to the interaction potential $V(r) . V(r)$ is characterized as the sum of a hardsphere potential $V_{\mathrm{hs}}(r)$, a repulsive screened Coulomb potential $V_{\mathrm{sc}}(r)$ and an attractive part, which is modeled as a Yukawian potential $V_{Y}(r)[8-10,15-18]$ :

$$
V(r)=V_{\mathrm{hs}}(r)+V_{\mathrm{sc}}(r)+V_{Y}(r),
$$

with

$$
\begin{aligned}
& V_{\mathrm{hs}}(r)= \begin{cases}\infty & r \leq \sigma \\
0 & r>\sigma\end{cases} \\
& V_{\mathrm{sc}}(r)= \begin{cases}0, r \leq \sigma \\
\frac{Z^{2} e^{2}}{4 \pi \varepsilon_{0} \varepsilon_{\mathrm{r}}(1+0.5 \kappa \sigma)^{2}} & \frac{\mathrm{e}^{-\kappa(r-\sigma)}}{r}, r>\sigma\end{cases} \\
& V_{Y}(r)= \begin{cases}0, r \leq \sigma \\
-J \sigma \frac{\mathrm{e}^{-(r-\sigma) / d}}{r}, & r>\sigma\end{cases}
\end{aligned}
$$

$e$ is the elementary charge, $\varepsilon_{0}$ the dielectric permittivity of the vacuum, $\varepsilon_{r}$ the dielectric number of the solution, and $\kappa$ is the reciprocal screening length. The pressure dependence of $\varepsilon_{r}$ and thus $\kappa$ was taken into account and can be found in [19]. Reasonable assumption of a constant effective protein charge of $Z=8$ at $p \mathrm{H} 7$ as determined by titration experiments [20], an effective protein hardsphere diameter of $\sigma=2.99 \mathrm{~nm}$, and fixing the $d$ value at $2.7 \AA$, the strength $J$ of the attractive part of $V(r)$ can be calculated from fits of the scattering data using the MSA approach [14,15].

The scattering pattern at the lowest concentration $(0.45 \mathrm{wt} \%)$ exhibits negligible interference effects and represents the form factor $P(q)$ of the particle. It is adequately described by the computed scattering form factor of a prolate ellipsoid of revolution with radius of gyration $R_{g}=(14.8 \pm 0.5) \AA$ and axial ratio of $1.5(V=$ $25.4 \mathrm{~nm}^{3}$ ), which is in good agreement with literature data [21]. Comparing the $P(q)$ values obtained for all pressures and temperatures studied, no differences due to changes of the tertiary structure of the protein were detected, indicating that the protein remains in its native, folded conformation at all conditions studied.

As depicted in Fig. 1(a), the SAXS intensity curves $I(q)$ of the concentrated protein solutions show a pronounced correlation peak due to the presence of a structure factor
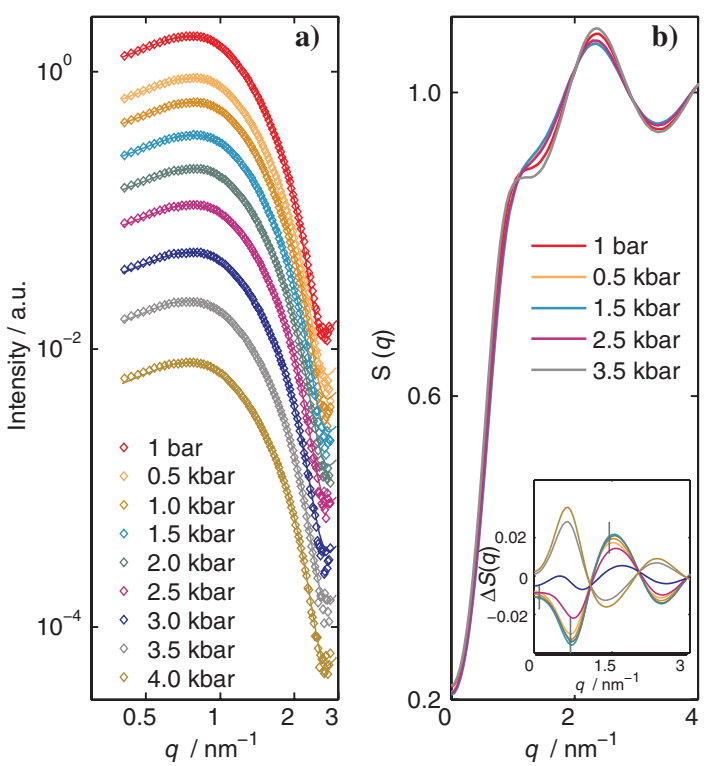

FIG. 1 (color). (a) Experimental SAXS data $I(q)$ of a lysozyme solution $\left(c_{p}=100 \mathrm{mg} \mathrm{mL}^{-1}\right)$ at $T=25^{\circ} \mathrm{C}$ for different pressures with corresponding fitting curves using the DLVO potential and MSA superimposed on the experimental data. (b) Structure factors $S(q)$ obtained for selected pressures. Inset (with representative maximum error bar): $\Delta S(q)=S(q)_{p^{-}} S(q)_{1 \text { bar }}$.

reflecting protein-protein interactions [7-10,22]. The structure factors $S(q)$, obtained by refinement of the scattering data $I(q)$, are shown in Fig. 1(b). To highlight the pressure-induced changes, the inset shows the differences $\Delta S(q)$ between $S(q)$ at high pressures and at 1 bar.

Increasing the hydrostatic pressure $p$ from 1 bar up to $1.5 \mathrm{kbar}$ leads to a shift of the correlation peak to larger $q$ values, i.e., smaller distances. Surprisingly, as the pressure is further increased, this shift is reversed. As the correlation peak is caused by the intermolecular structure factor $S(q)$, differences in the region below and above $1.5 \mathrm{kbar}$ are more pronounced in $S(q)$ as depicted in Fig. 1(b). This effect is more visible in the $\Delta S(q)$ data, which change sign in the intermediate pressure range [inset of Fig. 1(b)]. For $p=1.5 \mathrm{kbar}$, the determined structure factor has a shape typical for largely repulsive systems [17]. At this pressure, the mean intermolecular $d$-spacing $d_{\text {inter }}\left(d_{\text {inter }} \approx\right.$ $2 \pi / q_{\max }, q_{\max }$ : first maximum of $\left.S(q)\right)$ has decreased from $\sim 57 \AA$ to $\sim 46 \AA \AA$ upon pressurization, and $d_{\text {inter }}$ reaches a value of $\sim 55 \AA$ at $4 \mathrm{kbar}$.

To verify that such pressures do not alter the protein's secondary structure, complementary FTIR measurements were carried out using a Nicolet MAGNA 550 spectrometer equipped with a liquid nitrogen cooled $\mathrm{MCT}(\mathrm{HgCdTe})-$ detector and a diamond anvil cell [23]. Analysis of the subbands in the conformation-sensitive amide I band region allows extraction of the secondary structure elements of the protein [23] (for details see the SI [24]). As can be seen in Fig. 2, the secondary structure of the protein remains unchanged up to about $5 \mathrm{kbar}$, where the protein 


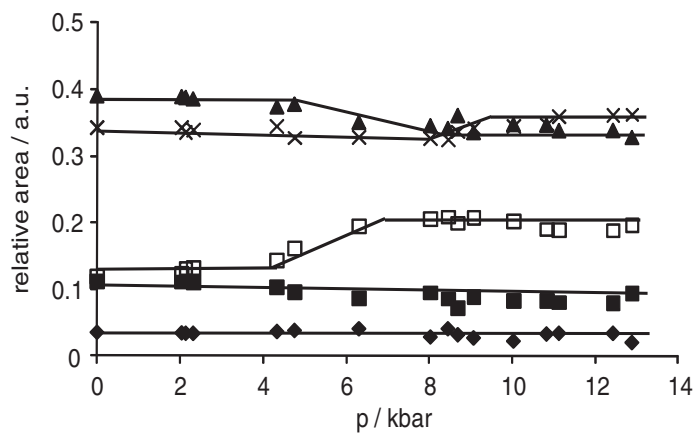

FIG. 2. Relative changes of secondary structure elements $(\boldsymbol{\Lambda}$, $\alpha$ helices; $X$, disordered structures; $\square, \beta$ strands; $\bullet$ and $\mathbf{\square}$ : turns/loops) as obtained from deconvolution of the amide I' band of FTIR spectroscopic data (see also [24]).

starts to partially unfold and rearrange, indicated by an increase in disordered structures and new $\beta$ conformations at the expense of $\alpha$-helical structures. These data confirm that in the pressure range where the SAXS measurements were carried out (up to 4 kbar), no significant changes in secondary structure occur; i.e., the SAXS changes observed are purely due to intermolecular interaction effects.

From the fits of the SAXS data [full lines in Fig. 1(a)] employing the liquid state physics approach described above, the pressure dependence of the strength of the attractive part of the interaction potential, $J$, could be determined for the different solution temperatures and pressures measured. As can be clearly seen in Fig. 3, the pressure dependence of $J$ looks similar for all temperatures: $J(p)$ first decreases with pressure, but then reveals a minimum at 1.5-2 kbar. As expected, decreasing the temperature results in an increase of $J$, i.e., an increase in intermolecular attraction of the proteins. The maximal error in $J$ is given by the direct comparison of the results of the SAXS data obtained on two different instruments.

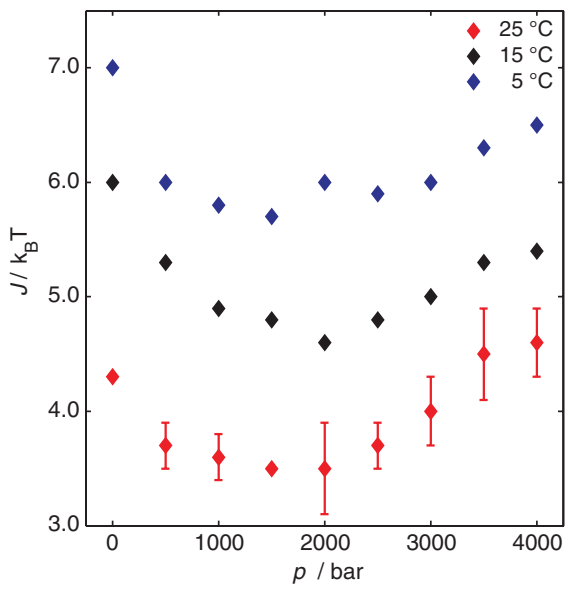

FIG. 3 (color). Experimentally determined strength $J$ of the attractive part of the interaction potential $V(r)$ as a function of pressure for three different temperatures. Also shown is the maximal error of the $J$ values.
Please note that with the simpler random phase approximation (RPA), a similar pressure dependence of $J$ is obtained.

The effect of pressure on the shape of the total intermolecular potential $V(r)$ for the highest and the lowest temperatures measured is shown in Fig. 4. For $T=$ $25^{\circ} \mathrm{C}$, the application of pressure up to $1.5 \mathrm{kbar}$ results in a slight increase of the repulsive part of $V(r)$ upon pressurization of the solution containing the positively charged protein. A further increase of pressure leads to a slight reduction of the repulsion barrier, accompanied by the slightly increased average intermolecular separation discussed above.

Decreasing the temperature down to $T=5{ }^{\circ} \mathrm{C}$ reduces the strength of the repulsive Coulomb potential, and $J$ increases continuously due to the increase of the depth of the attractive part of $V(r)$. The influence of pressure on $J(p)$ and hence $V(r)$ is similar at the lower temperature. As the particle density changes by $\sim 14 \%$ only upon pressurization up to $4 \mathrm{kbar}$ at these temperatures, the pressure effect on $J$ is much smaller than the temperature effect. A similar scenario has also been observed for higher protein concentrations (e.g., $190 \mathrm{mg} \mathrm{mL}^{-1}$, see [24]).

In the absence of protein unfolding and conformational changes, and after having taken into account the pressure dependence of the bulk properties of the solvent [such as $\varepsilon_{r}(p)$ and $\left.\kappa(p)\right], V(r)$ can still be influenced by pressure through changes in the hydration properties. In fact, kbarpressures modify the structure of liquid water, which is also reflected in changes of the transport properties, such as the viscosity and diffusion coefficent, which exhibit changes in their pressure coefficents around 2 kbar [25]. The pressure-induced volume reduction is accompanied by a marked change in local molecular arrangement below 5 kbar. Whereas the distance of nearest neighbors $r_{1}$
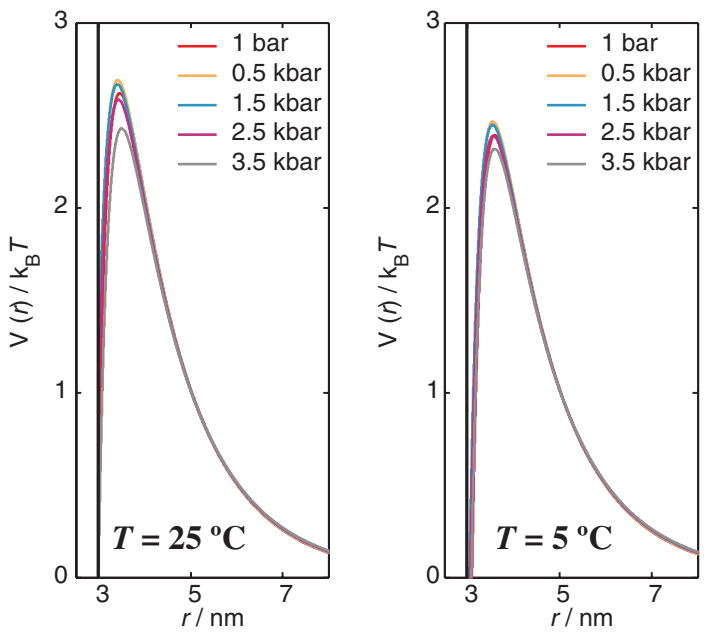

FIG. 4 (color). Total interaction potential $V(r)$ of a $10 \mathrm{wt} \%$ lysozyme solution at $p \mathrm{H} 7$ and selected pressures for two temperatures. The black solid line represents the impenetrable hard core contribution. 
remains essentially the same upon pressurization, the volume reduction is caused by a rapid and monotonic increase in the coordination number $N_{1}$, from $\sim 4$ to 6.5 at $5 \mathrm{kbar}$, which can be attributed to the penetration of nonhydrogenbonded water molecules into the first shell [26-28]. These structural changes in the kbar pressure range seem coincide with the observed nonlinear pressure response of $V(r)$. At pressures below $\sim 2.0 \mathrm{kbar}$, pressure leads to a reduction of the intermolecular distances resulting in a decrease of $J$. Beyond this pressure threshold, the high-density structure and concomitant increased hydration repulsion of water may lead to the effective small increase of $J$ and the slight increase of intermolecular distances observed.

To summarize, SAXS patterns have been recorded of dense lysozyme solutions at selected temperatures in a pressure range up to $4 \mathrm{kbar}$. The scattering patterns could be fitted by considering systems of individual lysozyme particles with an effective interaction potential consisting of a short-range attraction and long-range repulsion (screened Coulomb) term. The interactions reported here concern the native protein in a crowded environment that does not undergo any significant conformational changes. The interaction parameters of these dense protein solutions are affected by pressure in a nonlinear way. At pressures of $\sim 2$ kbar (where intermolecular distances have decreased by about 6\%, and intermolecular separations reach $\sim 2.7 \mathrm{~nm}$, corresponding to an average of $\sim 9$ water layers), i.e., the pressure regime where the coordination number of water has increased markedly due to a collapsed second hydration shell, protein-protein interactions are slightly modified. The structural changes of the solvent lead to a modification of the interparticle repulsion and hindrance of closer approach of the proteins at higher pressures, thus preventing them from aggregation. For higher concentrated solutions, the pressure where this trend reversal occurs is found at similar pressures, around $2 \mathrm{kbar}$ (see [24]).

These findings are thus also of importance for understanding the high stability of dense protein solutions (as they occur intracellularly) in biosystems living under hydrostatic pressure conditions such as in the deep sea. Moreover, these studies may also prove important for exploring largely untouched research areas, such as the effect of pressure on nucleation and crystal growth phenomena of proteins, on protein aggregation, and for understanding the complex phase behavior of dense protein solutions in general [29-31]. In all these cases, the particular structural properties of water might influence the spatial organization of the proteins at high-pressure conditions.

This research was supported by the DFG.
[1] P. W. Bridgman, J. Biol. Chem. 19, 511 (1914).

[2] J. L. Silva, D. Foguel, and C. A. Royer, Trends Biochem. Sci. 26, 612 (2001).

[3] F. Meersman, C.M. Dobson, and K. Heremans, Chem. Soc. Rev. 35, 908 (2006).

[4] K. Akasaka, Chem. Rev. 106, 1814 (2006).

[5] R. Winter, D. Lopes, S. Grudzielanek, and K. Vogtt, J. Non-Equilib. Thermodyn. 32, 41 (2007).

[6] R. J. Ellis, Trends Biochem. Sci. 26, 597 (2001).

[7] M. G. Ortore et al., J. R. Soc. Interface 6, S619 (2009).

[8] J. Narayanan and X. Y. Liu, Biophys. J. 84, 523 (2003).

[9] N. Javid, K. Vogtt, C. Krywka, M. Tolan, and R. Winter, Chem. Phys. Chem. 8, 679 (2007).

[10] N. Javid, K. Vogtt, C. Krywka, M. Tolan, and R. Winter, Phys. Rev. Lett. 99, 028101 (2007).

[11] R. C. Neuman et al., J. Phys. Chem. 77, 2687 (1973).

[12] C. Krywka, C. Sternemann, M. Paulus, M. Tolan, C. Royer, and R. Winter, Chem. Phys. Chem. 9, 2809 (2008).

[13] M. Kotlarchyk and S.-H. Chen, J. Chem. Phys. 79, 2461 (1983).

[14] Y. Liu, W.-R. Chen, and S.-H. Chen, J. Chem. Phys. 122, 044507 (2005).

[15] S. Longeville, W. Doster, and G. Kali, Chem. Phys. 292, 413 (2003).

[16] M. Malfois et al., J. Chem. Phys. 105, 3290 (1996).

[17] F. Zhang et al., J. Phys. Chem. B 111, 251 (2007).

[18] V. K. Kelkar, J. Narayanan, and C. Manohar, Langmuir 8, 2210 (1992)

[19] W. B. Floriano and M. A.C. Nascimento, Braz. J. Phys. 34, 38 (2004).

[20] D.E. Kuehner et al., J. Phys. Chem. B 103, 1368 (1999).

[21] A. Shukla et al., Proc. Natl. Acad. Sci. U.S.A. 105, 5075 (2008).

[22] A. Stradner, H. Sedgwick, F. Cardinaux, W. C. K. Poon, S. U. Egelhaaf, and P. Schurtenberger, Nature (London) 432, 492 (2004).

[23] S. Grudzielanek, V. Smirnovas, and R. Winter, J. Mol. Biol. 356, 497 (2006).

[24] See supplemental material at http://link.aps.org/ supplemental/10.1103/PhysRevLett.106.178102.

[25] R. Ludwig, Angew. Chem., Int. Ed. 40, 1808 (2001).

[26] A. K. Soper and M. A. Ricci, Phys. Rev. Lett. 84, 2881 (2000).

[27] G. Weck et al., Phys. Rev. B 80, 180202(R) (2009).

[28] Y. Katayama et al., Phys. Rev. B 81, 014109 (2010).

[29] R. Mishra and R. Winter, Angew. Chem., Int. Ed. 47, 6518 (2008).

[30] A. Stradner, G.M. Thurston, and P. Schurtenberger, J. Phys. Condens. Matter 17, S2805 (2005).

[31] M. Muschol and F. Rosenberger, J. Chem. Phys. 107, 1953 (1997). 